\title{
A neuromarker for drug and food craving distinguishes drug users from non-users
}

\author{
Leonie Koban ( $\nabla$ Leonie.Koban@gmail.com ) \\ CNRS https://orcid.org/0000-0002-3121-6491 \\ Tor Wager \\ Dartmouth College https://orcid.org/0000-0002-1936-5574 \\ Hedy Kober \\ Yale University
}

Article

Keywords: craving, brain signature, drug use

Posted Date: August 9th, 2021

DOI: https://doi.org/10.21203/rs.3.rs-726333/v1

License: (a) This work is licensed under a Creative Commons Attribution 4.0 International License. Read Full License

Version of Record: A version of this preprint was published at Nature Neuroscience on December 19th, 2022. See the published version at https://doi.org/10.1038/s41593-022-01228-w. 


\section{Abstract}

Craving is a core feature of substance use disorders and a strong predictor of relapse. It is linked to polysubstance use, overeating, pathological gambling, and other maladaptive behaviors. Despite its utility, craving measures are based on self-report, with associated limitations related to introspective access and variability across sociocultural contexts. Objective biological markers of craving, which could reveal the neurophysiology of craving, are lacking. For example, it remains unclear whether craving for drugs and food involve similar or separable mechanisms. Across three studies $(N=101)$, we combined fMRI with machine-learning to identify a brain-based marker of cueevoked drug and food craving, resulting in a cross-validated multi-system pattern (or brain signature), including ventromedial prefrontal and cingulate cortices, ventral striatum, temporal and parietal association areas, mediodorsal thalamus, and cerebellum. This signature predicted self-reported craving intensity $(p<0.002$, within-person $r=0.50)$ and discriminated high from low craving with $78 \%$ accuracy. It also discriminated drug users versus non-users based on brain responses to drug (75\% accuracy), but not food, cues. Predictive brain models trained on drug cues also transferred to food cues, and vice versa, suggesting shared neurophysiological mechanisms for drug and food craving. In conclusion, fMRI can be used to predict craving across different drug and food cues, and separate drug users from non-users. Future studies are needed to assess whether the brain signature of craving responds to clinical intervention and can predict long-term clinical outcomes.

\section{Introduction}

Substance use and unhealthy eating contribute to the top three preventable causes of disease and death [1,2]. Substance use disorders (SUDs) are among the most prevalent and deadly psychiatric conditions worldwide [3]. In the US alone, the economic costs associated with the use of tobacco, alcohol, and illicit drugs amount to more than $\$ 740$ billion per year [4]. Further, SUDs are associated with staggering social costs and personal suffering. Nearly 70,000 people died of drug overdose in the US in 2018 [5]. More than 480,000 deaths in the US each year are attributed to smoking [6], and over 90,000 deaths are attributed to alcohol use [7]. Obesity, a related biobehavioral risk factor, affects $42 \%$ of adults in the US [6] and its prevalence is increasing worldwide [8]. It is associated with heart disease, diabetes, and other disorders, contributing to $18 \%$ of annual deaths [9] and medical costs of $\$ 147$ billion US dollars per year in the US [10]. Thus, understanding the biological and psychological contributors of drug use and overeating is of tremendous importance.

Craving - a strong urge to use drugs or to eat-has long been considered to be a core factor driving unhealthy eating and substance use [11]. In 2013, it was added as a diagnostic criterion for SUDs in the DSM-5 [12]. In particular, cue-induced craving, which arises in response to drugor food-related stimuli, is known to prospectively predict eating, weight gain, drug use, and relapse [13-15]. Because it is a common predictor across multiple disorders and maladaptive behaviors, it may constitute a transdiagnostic risk factor.

While self-reported craving has been useful clinically as well as experimentally, there is growing recognition of the need to understand its biological basis. Human neuroimaging studies have identified circuits related to substance use risk, incidence, and sequelae [e.g., 16, 17-21]. Some, including ventromedial prefrontal (vmPFC)-ventral striatal/accumbens (VS/NAc) circuits, are associated across substance use disorders, outcomes, and risky behaviors, and have been supported by converging evidence from animal models [22-28]. This circuit and others can be targeted by neurostimulation, pharmacological, psychological, and behavioral interventions [28-30].

Though great strides have been made in our understanding of substance misuse and related phenomena, our understanding of the neural basis of craving is lacking. While the neuroimaging literature on craving is growing, craving cannot be directly measured in nonhumans (cf. [31]). In addition, understanding that any specific brain region is involved in craving or other outcomes does not imply that we can decode craving from the brain, or that we have a sufficiently precise measurement model to allow for the prediction of outcomes [32, 33]. It is increasingly apparent that many mental states and related outcomes have a highly distributed brain basis [34, 35], including emotion [35-38], pain [35, 36, 39-43], perception [44-46], object recognition [47-50], memory retrieval [51, 52], sustained attention [53, 54], semantics [47, 55, 56], and autonomic responses [57]. Accordingly, measures that integrate across brain systems can provide sensitive, specific, and generalizable characterizations of the neurophysiological underpinnings of behavior [58]. They can also predict health-related outcomes with larger effect sizes than measures based on single regions in many cases [59].

Predictive models-also called 'neuromarkers' or 'signatures'-have multiple potential uses [60-62]. They can predict risk for future disorders, identify subtypes (or biotypes) that predict who will respond to a treatment, and perhaps most importantly serve as mechanistic targets for interventions. They can also outperform subjective measures in predicting human choices [63], and can be linked with systems and cellular neuroscience to develop new biological treatments in 'reverse translation' approaches [31]. Accordingly, there is increasing recognition of the need to develop biomarkers based on human systems that can be compared with animal models $[61,62,64,65]$. However, such approach has rarely been applied in addiction [66], and has not yet been applied to craving. 
Here, we take a first step towards a neuromarker that predicts the intensity of craving in clinical and matched control samples. We integrated data from 5 different cohorts in $3 \mathrm{fMRI}$ studies across different types of drug users (alcohol, cocaine, cigarettes), and non-users (total of 479 contrast images from $\mathrm{N}=101$ participants). Across studies, participants were presented with visual cues of drugs and highly palatable food items. We then used machine-learning to identify a distributed functional brain activity pattern that predicted the intensity of craving.

We term the resulting pattern the Neurobiological Craving Signature (NCS), and we hope that this name would reduce ambiguity and provide a reference point for the pattern's future reuse and testing in new samples. Analyses related to the NCS allow us to address scientific questions related to the organization of craving-related brain systems across drugs and food (or other rewarding stimuli), and their susceptibility to cognitive, pharmacological, and other interventions. Further, recent perspectives have proposed a common neurophysiology for SUDs and obesity, and of drug and food craving more specifically [67-71], but this view has been challenged [72, 73]. The NCS allows us to test whether craving for several types of legal and illicit drugs and for highly palatable foods are based on different or shared neurophysiological patterns. We further assess whether the brain systems involved in cue-induced craving are affected by cognitive regulation strategies, highlighting the malleability of craving-related brain patterns to interventions and thus opening avenues for developing further interventions and improving existing ones.

\section{Results}

\section{Data overview}

A total of 479 contrast images from 101 participants and 5 independent cohorts were used for training and testing the pattern to predict drug and food craving (two drug using cohorts, two of their matched controls, and another sample of drug users with no matched controls). All participants viewed images of drugs and food under two instructions conditions: a craving instruction and an instruction to use a cognitive strategy to reduce craving (see methods; [74-76]. Contrast images were computed for the onset of the visual drug and food cues (see Figure 1a), separately for each level of craving (1-5 Likert scale) for every participant.

\section{fMRI results}

Description of the Neurophysiological Craving Signature (NCS). Parallel to previous studies on fMRI-based prediction of pain and emotion [36, 39, 77, 78], LASSO-PCR and study-stratified 10-fold cross-validation was used to predict the level of craving based on fMRI contrast images. Figure 2 presents a thresholded display of the resulting weight map based on bootstrapping. While the unthresholded map is used for prediction, the thresholded map illustrates the brain areas that most robustly contribute positive or negative weights to the predictive pattern. Areas with positive weights included ventromedial prefrontal cortex, dorsal anterior cingulate cortex, subgenual cingulate/ventral striatum, retrosplenial cortex, parietal and temporal areas, cerebellum, and amygdala. Negative weights were found in visual areas, lateral prefrontal, parietal and somatomotor areas, among others (see Table 1 for a list of FDR-corrected coordinates). Of note, many areas, including somatomotor cortex, parietal, temporal, and bilateral insula included clusters of both positive and negative weights.

Predictive performance of the NCS. The trained pattern resulted in a cross-validated prediction-outcome correlation of $r=0.50$ (S.D. +-0.49) within-person and $r=0.33$ across all data points, with a mean absolute error of 1.30 points on the 1-5 Likert scale. A multi-level general linear model (GLM) confirmed a strong relationship between out-of-sample predicted and actual level of craving with a large effect size (beta = 0.35 , STE $=0.04, t(100)=8.43, p<0.00001$, Cohen's $d=0.84)$. The strength of the predictive performance varied across datasets, but was significant in all 5 cohorts, with effect sizes (Cohen's $d$ ) ranging from 0.59-1.38 (see Table 2).

Classification of high versus low craving. We next assessed the accuracy of this pattern to differentiate between high versus low levels of craving. Forced-choice binary classification of highest versus lowest levels of craving per participant was achieved with a cross-validated accuracy of $78 \%+-4.1 \%$ STE, binomial test $p<0.00001$ (sensitivity $=78 \%$, specificity $=78 \%$, area under the curve [AUC] $=0.89$ ). Even across subjects (single-interval classification), this pattern separated brain responses to the highest versus lowest individual levels of craving with $72 \%$ cross-validated accuracy (+-3.3\%STE, binomial test $p<0.00001$, sensitivity $=65 \%$, specificity $=78 \%, A U C=0.75)$. While this level of predictive accuracy does not provide perfect separation of high versus low craving, it is remarkable, since all stimuli were drugs or highly palatable food items, thus differences in classification performance were not driven by external stimulus characteristics but by the personal history and internal motivational states of the participants. 
Differentiating drug users from non-users. We next tested whether individual craving-pattern responses to drug and food cues could be used to predict whether a participant was a drug user or non-user (see Figure 4a for group averages, Figure $\mathbf{4 b}$ for individual effects, and Figure 4c for ROC plots). While pattern expression in brain responses to food cues were not significantly differentiating drug users from non-users (59\% accuracy $+-4.9 \% \mathrm{STE}, P=0.92$, AUC $=0.41$ ), NCS pattern responses to drug cues significantly classified drug users from non-users, with $75 \%$ accuracy $(+-4.3 \%$ STE, $P=0.000616$, sensitivity $=86 \%$, specificity $=60 \%$, AUC $=0.76)$. When testing a pattern based on the drug $>$ food contrast, the response in the NCS separated drug users from non-users with $77 \%$ accuracy $(+-4.2 \%$ STE, $P=0.000113$, sensitivity $=86 \%$, specificity $=64 \%$, AUC $=0.83$, see Figure $4 c$ ).

Given slight but significant differences in years of education between users and non-users, we tested whether differences in NCS response could be explained by years of education. Individual differences in years of education correlated negatively with NCS response to drug cues $(r=-0.18, p=0.079)$ and to the contrast drug $>$ food cues $(r=-0.29, p=0.004)$. However, these associations did not mediate the relationship between drug-use status and NCS responses $(p=0.35)$ and were not significant when controlling for drug use $(p=0.32)$. Further, NCS responses to food cues did not correlate with years of education $(r=0.05, p=0.612)$. These results speak against the interpretation that NCS differences between drug users and non-users are driven by sociodemographic differences such as differences in education, and rather more likely reflect the fact that drug use (especially illegal drug use) reduces educational achievements [79].

Drug and food craving are predicted by shared brain patterns. An important debate concerns the question whether drug and food craving are based on similar brain processes $[67,68,70,73]$. If drug and food craving are driven by shared brain processes, then drug craving should be predictable based on a pattern that is trained to predict food craving, and food craving should be predictable based on a pattern that is trained to predict drug craving - at least in drug users. Conversely, if drug and food craving are based on dissociable brain processes, then better predictive accuracy will be gained by training drug- and food-specific (compared to craving-general) brain patterns.

We therefore repeated the procedures described above and tested whether training on drug and food data separately would improve prediction of craving, and whether food craving could be predicted based on a pattern trained on drug data only, and vice versa (Figure 5). Food craving was predicted similarly well by the overall pattern (75\% out-of-sample accuracy) as by a craving pattern trained on food cues only $(78 \%)$. Food craving was also significantly predicted by a pattern trained on drug cues only, but with somewhat lower accuracy across both drug-using and non-using participants $(62 \%)$. For the prediction of drug craving, the results indicated no substantial improvements for training only on modality-specific (drug) cue trials (69\% out-of-sample prediction accuracy) compared to all cues (66\%). Drug craving was also significantly predicted by a pattern that was trained to predict food craving (63\% out-of-sample predictive accuracy) to training only on food trials. Thus, we did not find evidence for a double dissociation between drug and food craving, but rather significant cross-prediction of drug and food craving. Most importantly, the NCS performed as well as the two cue-specific patterns. Together, this supports the hypothesis of shared representations between drug and food craving - and across drug types.

Modulation by cognitive regulation strategies. Finally, we used a general linear model to assess how craving ratings and responses of the NCS were modulated by the cognitive regulation of craving (ROC) task that was employed in all five studies. Across all participants, craving ratings were influenced by cue type (drug vs. food, $F_{(1,396)}=72.6, p<0.001$ ) and regulation instruction (NOW vs LATER, $F_{(1,396)}=92.4, p<$ 0.001). Drug users reported greater overall craving $\left(F_{(1,396)}=56.4, p<0.001\right)$ and this effect interacted with both regulation instruction $\left(F_{(1,}\right.$ $396)=5.8, p=0.017)$ and cue type $\left(F_{(1,396)}=151.0, p<0.001\right)$, such that drug users craved drugs more than non-users, and that they showed slightly higher regulation effects than non-users. This was due to the fact that non-users had very low craving for drug cues, irrespective of regulation condition (see Figure 3d). In line with this 'floor effect', there was also a significant interaction between drug-use, cue type, and regulation condition $\left(\mathrm{F}_{(1,396)}=20.3, p<0.001\right)$.

Similar to craving ratings, responses of the NCS were influenced by cue type (drug vs. food, $F_{(1,396)}=71.9, p<0.001$ ) and by regulation instruction (NOW vs LATER, $F_{(1,396)}=43.2, p<0.001$ ), suggesting that cognitive regulation strategies modify NCS responses. Drug users versus non-users had marginally greater NCS responses overall $\left(F_{(1,396)}=3.4, p=0.067\right)$. Drug users' versus non-users' signature response differed with respect to cue type $\left(F_{(1,396)}=56.9, p<0.001\right)$, such that drug users had higher NCS responses to drug cues than non-users $(t(99)=4.40, p<0.001)$, whereas NCS responses to food cues did not significantly differ $(t(99)=-1.07)$. Further, drug users' versus non-users' signature response differed with respect to regulation condition $(F(1,396)=6.9, p=0.009)$, such that users showed a larger regulation effect that non-users $(t(99)=2.63, p=0.0099$, see Figure $2 \mathbf{e})$, which was likely driven by more room to down-regulate craving in users compared to non-users. 


\section{Discussion}

Craving is arguably one of the most central processes in SUDs [15]. It contributes to multiple behaviors that are detrimental to physical and mental health in the long term, including smoking, alcohol drinking, overeating, and gambling [13-15]. As other transdiagnostic key processes - and human behavior more broadly - craving results from brain function. However, it is typically assessed using subjective measures that require introspection and are sensitive to context; thus, there is a strong need for biomarkers, and particularly neuromarkers based on brain function $[32,64,66,80,81]$. Such biomarkers can identify mechanistic targets that can aid in monitoring disease progression (monitoring biomarkers according to the FDA), identifying individuals at risk for SUDs and future weight gain (prognostic biomarkers), predicting treatment response (predictive biomarkers), and serving as targets for neuromodulatory and behavioral interventions [62].

Here we used machine learning to identify a distributed brain pattern - which we term the NCS as a reference for future use - that tracks the degree of craving when applied to new individuals, across different diagnostic groups, scanners, and scanning parameters. Importantly, this pattern separated drug users from non-users based on brain responses to drug cues, but not food cues. Thus, it is a crucial first step towards a diagnostic neuromarker of substance use. Further, given the role of self-reported craving in predicting outcomes, this brain-based pattern may function as both a diagnostic and predictive biomarker with potential utility in predicting clinically relevant individual differences and future outcomes. Future studies could build on these findings to test whether the NCS responds to therapeutic interventions that reduce craving and/or drug use and whether it has predictive value for long-term clinical outcomes such as drug relapse or weight gain. Further, our finding that the NCS is sensitive to cognitive regulation strategies suggests that it may be psychologically modifiable. This is important because psychological and behavioral interventions can be effective, but their mechanisms are poorly understood. Furthermore, current interventions are associated with high rates of relapse and could be improved [82]. Future models could also be developed based on other data types (e.g., resting-state fMRI, imaging in animals) or their combination [32, 66].

Our results also offer new insight into a long-standing debate concerning the question whether craving of drugs of abuse and food share common underlying brain functions, especially in motivation-related circuits [32]. We show that craving of various types of drugs and food can be predicted by largely shared whole-brain activity patterns. Indeed, the results demonstrate that craving-related responses to cues for legal and illegal drugs and for highly palatable food items are surprisingly similar and not dissociable at the fMRI pattern level in both drug users and non-drug using adults. This is noteworthy, especially as the non-users in the present studies were not obese or "food addicted," but rather healthy controls. Importantly, this overlap is consistent with models suggesting that drug craving depends on systems evolved for seeking highly palatable food and other primary rewards [68]. Future research could test whether the NCS also responds to less palatable or healthy food items, and to other types of primary and secondary rewards.

Some areas in the NCS, including the vmPFC and ventral striatum/accumbens, have been broadly implicated in reward and valuation [83, 84], and have long been associated with craving and substance use across species. A number of prior studies and several meta-analyses [71, 85-90] have demonstrated a central role of VMPFC, VS, amygdala, insula, and posterior cingulate cortex in drug and food cue reactivity and craving (although findings across meta-analyses are inconsistent). The vmPFC has been targeted in rTMS studies to successfully reduce drug craving [17]. The positive peaks of the NCS in this area could thus serve as a more precise target for neurostimulation. Future studies can test whether successful neurostimulation of VmPFC also reduces NCS expression and alters connectivity of the vmPFC with other NCS core areas such as the ventral striatum.

The insula is connected to many regions of the NCS and has been previously associated with craving [91]. Lesions in various insular locations have been shown to reduce the urge to use drugs and facilitate smoking cessation [92], which could reflect the role of the insula in the interoceptive component of drug craving [92-94]. The NCS has positive weights (at uncorrected thresholds) in the mid and posterior insula, in line with these previous reports. However, the anterior insula also displayed negative weights in the NCS (at uncorrected thresholds), revealing a potentially more complex role of different insula subregions in craving. Further, the insula might be more prominent to bodily cues of withdrawal, craving, and negative affect [91, 95], as well as for nutrient-related reward signals [96], whereas areas such as amygdala or vmPFC (which are more prominent in the NCS) are related to craving evoked by external cues [95] such as those employed in the present datasets.

The NCS further extends prior work in several ways: First, it includes strong positive and negative weights in brain areas not previously associated with craving, such as the cerebellum, lateral temporal, and parietal areas. These areas are connected to regions more traditionally associated with craving and might constitute new targets for investigation and intervention. Second, the NCS is a precise and replicable pattern, including relative activity levels across voxels within key regions and relative activity across networks. Thus, it constitutes a reproducible brain model $[58,97]$ of craving that can be empirically quantified and validated in any new brain imaging study or dataset.

The present findings have some limitations that could be addressed in future studies. First, the included studies used a limited set of highly appetitive cues. Future studies could use a larger range of stimuli, including less palatable (and healthier) food items or non-craving related 
(neutral) cues. Greater variation in craving ratings should - in principle - lead to increased discrimination accuracy between low and high craving. Future studies could also test other modalities of drug and food cues (such as cigarette smoke or food smells, videos). In addition, fMRI has an inherently limited spatial resolution that cannot pinpoint the cellular or microstructural processes associated with craving, or different types of craving. However, craving cannot be directly assessed in animals and this work fills a crucial gap across species and brain systems. and is important for clinical uses in humans as well as other translational applications of MRI-based neuromarkers, which will inevitably use different scanners, hardware, and processes that evolve over time-thus requiring a focus on large-scale patterns that are generalizable across studies, scanners, groups, different preprocessing protocols, and other factors.

In both Western and Eastern philosophy, craving has been considered a source of suffering and unhappiness. While craving is an important feature of SUDs, eating disorders, and other psychiatric conditions, it is also a general aspect of human experience. Identifying the neurobiological basis of this important driver of human behavior is thus an important step in mapping brain circuits to basic affective and mental processes. In this paper we introduce the NCS - the first fMRI-based neuromarker of drug and food craving, which classifies drug users from non-users based on responses to drug, but not food cues. As such, it offers a promising target for future research and clinical interventions.

\section{Method}

\section{Participants}

The present analysis used the pooled data from $\mathrm{N}=101$ participants (33 female, $\mathrm{M}_{\mathrm{AGE}}=34.1$ years, $\mathrm{SD}_{\mathrm{AGE}}=10.8$ ) collected across three independent neuroimaging studies (five different participant groups, three scanners, see Figure 1 and Table 3). Detailed methods and procedures as well as other results from these data sets, focusing on the effects of regulation on behavior and univariate brain responses, are reported elsewhere [74-76].

Analyses reported here were not reported previously, and the three studies have not been previously combined. Across studies, participants were recruited using flyers and ads (in newspapers, online bulleting boards, etc.) from communities around Yale and Columbia Universities. Participants were included in drug using groups $\left(\mathrm{N}=59, \mathrm{M}_{\mathrm{AGE}}=34.6\right.$ years, $\mathrm{SD}_{\mathrm{AGE}}=11.2,18$ female) based on verified clinical measures (e.g., structured clinical interviews for diagnosis and/or Fagerström test of nicotine dependence). Individuals were included in "healthy control" groups $\left(\mathrm{N}=42, \mathrm{M}_{\mathrm{AGE}}=33.4\right.$ years, $\mathrm{SD}_{\mathrm{AGE}}=10.5,16$ female) if they were (1) age-, sex-, and race-matched to the SUD group in each respective study, (2) did not qualify for any SUD diagnosis or primary psychiatric diagnoses, and (3) did not regularly consume the substance of the SUD group in each respective study (i.e., matched healthy controls for the alcohol-using group did not regularly consume alcohol). Drug users did not significantly differ from non-users in age, sex, or racial/ethnic background. Compared to non-users, drug (especially cocaine) users had significantly lower years of education (see Table 3, 15.5 vs. 14.0 years, $p<0.001$ ). We therefore checked that the resulting neurobiological craving signature (NCS) was not related to education level above and beyond drug use status.

All participants provided informed consent. The studies have been approved by the institutional review boards of Columbia and Yale universities, and were conducted in compliance with all relevant ethical regulations.

\section{Regulation of Craving (ROC) task}

The ROC task is designed to evoke cue-induced craving of drug and food stimuli and test participants' ability to regulate craving [76]. Participants were shown images of drugs and food that were known to induce craving. On each trial, participants were instructed to observe these images in one of two ways. The NOW condition served as a craving baseline, whereby participants were instructed to consider the immediate positive consequences of consuming the pictured drug or food. In the LATER condition, participants were instructed to employ a cognitive strategy drawn from cognitive-behavioral treatments for substance use and obesity, and to consider the negative consequences of repeated consumption of the drug or food.

On each of 100 trials (50 drug, 50 food trials, presented in random order), participants were presented with a 2-second instructional cue (NOW or LATER) followed by a 6-second presentation of the drug or food image. After a jittered delay period (around 3 second), participants indicated how much they craved the drug or food at that moment ("How much do you want this?") on a 1-5 Likert scale, on which 1 indicated the lowest ("not at all") and 5 the highest ("very much") level of craving. Trials were separated by a jittered intertrial interval around 4 s, during which a fixation cross was displayed. Prior work [98-100] including the results from the pooled datasets [74-76] have confirmed that participants report less craving for food and drugs in the regulation (LATER) compared to the craving (NOW) condition. 


\section{fMRI data acquisition and preprocessing}

Data were collected on three different scanners at Columbia and Yale Universities using different acquisition parameters. Data underwent standard preprocessing in SPM (versions 5, 8, 12) including slice time correction, realignment, motion correction, warping, and smoothing with a $6 \mathrm{~mm}$ FWHM kernel. Differences in acquisition and preprocessing across datasets are in fact helpful in the current context as they ensure that our pooled findings are not dependent on such details.

\section{fMRI single trial models}

For each participant, we first computed a first level general linear model (GLM) using SPM8 and custom scripts (canlab.github.org). These models contained separate regressors for trials in the same condition and rating level (1-5), per run (modeled at 8s duration each). One additional regressor was added to model activity related to ratings (3s) across all trials. Further, 24 movement regressors (estimates for displacement and rotation in three dimensions, their derivatives, squared movement estimates, and derivatives of squared movement estimates) and spike regressors (based on the identification of global outliers, coded as 1 for the outlier time point and zero for all other time points) were added as regressor of no interest to control for motion artifacts.

Next, we averaged the resulting beta-images for each participant within each rating level. This resulted in up to five beta images per participant that reflected craving levels from 1 to 5 , respectively (if a participant did not have any ratings at a given level, a map for that level was not created for that participant). In order to bring all images to the same scale (thus increasing comparability across studies and scanners) and reduce the impact of potential outliers, L2norming was applied to all averaged beta images. An inclusive gray-matter-masks was applied to exclude voxels that likely contain white matter or cerebrospinal fluid only.

\section{Training and cross-validation of the NCS}

The resulting images for each level of craving were then used for linear prediction of craving using LASSO-PCR (least absolute shrinkage and selection operator-principal component regression) [101] and default parameters (to avoid overfitting). LASSO-PCR is a machine-learning algorithm that is well suited for prediction of outcomes based on large feature sets such as whole brain imaging data, which is characterized by substantially higher number of potential predictive features (i.e., voxels) than outcome data points (e.g., rating levels by subjects), and by a non-independence of these features (i.e., voxel activity is strongly covaried across regions and functional networks). LASSO-PCR avoids overfitting by first performing data reduction using principal component regression, thereby identifying brain networks that are characterized by high covariation of voxels. It then performs the LASSO algorithm, which reduces the contribution of less important or more unstable components by shrinking their regression weights towards zero. Voxel weights can be reconstructed based on their scores for the different components, thus rendering the resulting classifier interpretable and applicable to new datasets.

We used a 10-fold cross-validation procedure to evaluate the predictive accuracy of the classifier. Thus, we divided the data into 10 folds that were stratified by studies. Beta images of any given participants (corresponding to all levels of craving) were always held out in the same fold. In each iteration, the classifier was trained on the remaining data and then tested on the hold out data by calculating predicted level of craving as the dot product of the trained NCS and each held out beta-image. This out-of-sample predicted level of craving was used to assess differences in NCS responses between low and high craving ratings, experimental conditions (instruction, cue type), and drug users versus controls. For training and testing of drug- and food-craving patterns separately, the same procedure was repeated, but only using either drug or food contrast images, respectively.

\section{Bootstrapping and thresholding}

To assess the voxels with the most reliable positive or negative weights, we performed a bootstrap test. 10,000 samples with replacements were taken from the paired brain and outcome data and the LASSO-PCR was repeated for each bootstrap sample. Two-tailed, uncorrected $p$ values were calculated for each voxel based on the proportion of weights above or below zero $[39,77]$. False Discovery Rate (FDR) correction was applied to $p$-values to correct for multiple comparisons across the whole brain.

\section{Permutation tests}


Statistical significance of the cross-validated prediction accuracy was assessed using permutation tests. In each of 5000 iterations, craving ratings within each cohort were randomly permuted and training and cross-validation was performed on the permuted data to establish a null-distribution for performance measures (mean square error, root mean square error, mean absolute error, prediction-outcome correlation). Observed performance measures were compared to these permutation-based null-distributions in order to obtain non-parametric $p$-values.

\section{Classification analyses}

We used binary receiver operating characteristic (ROC) plots to illustrate the ability of the NCS to separate high versus low levels of craving using forced-choice tests (Figure 2), where pattern-expression values (the dot-product of the hold-out beta-images with the classifier weights) were compared for each participant's highest and lowest level of craving and the higher value was chosen as highest level of craving. To separate drug users from non-users (Figure 4b), pattern-expression values (separately for drug, food, or drug>food contrasts) or each participant were submitted to a single-interval test, thresholded for optimal overall accuracy. Area under the curve (AUC) is provided as a thresholded-independent measure of classification performance. Binomial tests were used to assess the statistical significance of classification accuracy.

\section{Declarations}

\section{Conflict of interest statement}

None of the authors have competing financial or non-financial interests.

\section{Data availability statement}

The datasets analyzed herein are available from Dr. Kober upon reasonable request.

\section{Code availability statement}

Matlab code for analyses is available at: https://github.com/canlab.

\section{References}

1. Janssen, F., S. Trias-Llimós, and A.E. Kunst, The combined impact of smoking, obesity and alcohol on life-expectancy trends in Europe. International Journal of Epidemiology, 2021.

2. Center for Disease Control and Prevention, National Health Report Highlights, U.S.D.o.H.a.H. Services, Editor. 2014: Atlanta, GA.

3. Suzuki, S. and H. Kober, Substance-Related and Addictive Disorders, in APA Handbook of Psychopathology. Vol. 1, Psychopathology: Understanding, Assessing, and Treating Adult Mental Disorders, J.N. Butcher, J. Hooley, and P.C. Kendall, Editors. 2018, American Psychological Association: Washington, DC. p. 481-506.

4. National Institute on Drug Abuse, Costs of Substance Abuse, N.I.o. Health, Editor. 2020: https://www.drugabuse.gov/drug-topics/trendsstatistics/costs-substance-abuse.

5. National Institute on Drug Abuse, Overdose death rates, N.I.o. Health, Editor. 2020: https://www.drugabuse.gov/drug-topics/trendsstatistics/overdose-death-rates.

6. Center for Disease Control and Prevention, Tobacco-Related Mortality, N.C.f.C.D.P.a.H. Promotion, Editor. 2020: https://www.cdc.gov/tobacco/data_statistics/fact_sheets/health_effects/tobacco_related_mortality/index.htm.

7. Center for Disease Control and Prevention, Deaths from Excessive Alcohol Use in the U.S., N.C.f.C.D.P.a.H. Promotion, Editor. 2020: https://www.cdc.gov/alcohol/features/excessive-alcohol-deaths.html.

8. World Health Organization, Obesity and overweight. 2020: https://www.who.int/news-room/fact-sheets/detail/obesity-and-overweight.

9. Masters, R.K., et al., The impact of obesity on US mortality levels: the importance of age and cohort factors in population estimates. Am. J. Public Health, 2013. 103(10): p. 1895-1901.

10. Center for Disease Control and Prevention, Adult Obesity Facts, N.C.f.C.D.P.a.H. Promotion, Editor. 2020: https://www.cdc.gov/obesity/data/adult.html. 
11. O'Brien, C.P., et al., Conditioning factors in drug abuse: Can they explain compulsion? Journal of Psychopharmacology, 1998. 12(1): p. 15-22.

12. American Psychiatric Association, Diagnostic and Statistical Manual of Mental Disorders - 5th Edition. 2013, Washington, DC: American Psychiatric Association.

13. Boswell, R.G. and H. Kober, Food cue reactivity and craving predict eating and weight gain: A meta analytic review. Obesity Reviews, 2016. 17(2): p. 159-177.

14. Vafay, N. and H. Kober, Cue reactivity and craving predict drug use and relapse: A quantitative meta-analysis. under review.

15. Sayette, M.A., The Role of Craving in Substance Use Disorders: Theoretical and Methodological Issues. Annu. Rev. Clin. Psychol., 2016. 12: p. 407-433.

16. Garavan, H., et al., Cue-induced cocaine craving: neuroanatomical specificity for drug users and drug stimuli. Am. J. Psychiatry, 2000. 157(11): p. 1789-1798.

17. Hanlon, C.A., et al., What goes up, can come down: Novel brain stimulation paradigms may attenuate craving and craving-related neural circuitry in substance dependent individuals. Brain Res., 2015. 1628(Pt A): p. 199-209.

18. Jasinska, A.J., et al., Factors modulating neural reactivity to drug cues in addiction: a survey of human neuroimaging studies. Neurosci. Biobehav. Rev., 2014. 38: p. 1-16.

19. Cassidy, C.M., et al., Evidence for dopamine abnormalities in the substantia nigra in cocaine addiction revealed by neuromelaninsensitive MRI. Am. J. Psychiatry, 2020. 177(11): p. 1038-1047.

20. Zubieta, J.-K., et al., Increased mu opioid receptor binding detected by PET in cocaine-dependent men is associated with cocaine craving. Nat. Med., 1996. 2(11): p. 1225-1229.

21. Heilig, M., et al., Addiction as a brain disease revised: why it still matters, and the need for consilience. Neuropsychopharmacology, 2021: p. 1-9.

22. Robinson, T.E. and K.C. Berridge, The neural basis of drug craving: an incentive-sensitization theory of addiction. Brain Res. Brain Res. Rev., 1993. 18(3): p. 247-291.

23. Berridge, K.C. and T.E. Robinson, Liking, wanting, and the incentive-sensitization theory of addiction. Am. Psychol., 2016. 71(8): p. 670679.

24. Kalivas, P.W., The glutamate homeostasis hypothesis of addiction. Nat. Rev. Neurosci., 2009. 10(8): p. 561-572.

25. Bedi, G., et al., Incubation of cue-induced cigarette craving during abstinence in human smokers. Biol. Psychiatry, 2011. 69(7): p. 708711.

26. Schoenbaum, G. and Y. Shaham, The role of orbitofrontal cortex in drug addiction: a review of preclinical studies. Biol. Psychiatry, 2008. 63(3): p. 256-262.

27. Everitt, B.J., A. Dickinson, and T.W. Robbins, The neuropsychological basis of addictive behaviour. Brain Res. Brain Res. Rev., 2001. 36(2-3): p. 129-138.

28. Lüscher, C., T.W. Robbins, and B.J. Everitt, The transition to compulsion in addiction. Nat. Rev. Neurosci., 2020. 21(5): p. $247-263$.

29. Ekhtiari, H., et al., Transcranial electrical and magnetic stimulation (tES and TMS) for addiction medicine: A consensus paper on the present state of the science and the road ahead. Neurosci. Biobehav. Rev., 2019. 104: p. 118-140.

30. Salling, M.C. and D. Martinez, Brain Stimulation in Addiction. Neuropsychopharmacology, 2016. 41(12): p. $2798-2809$.

31. Venniro, M., et al., Improving translation of animal models of addiction and relapse by reverse translation. Nature Reviews Neuroscience, 2020. 21(11): p. 625-643.

32. Whelan, R., et al., Neuropsychosocial profiles of current and future adolescent alcohol misusers. Nature, 2014. 512(7513): p. $185-189$.

33. Whelan, R. and H. Garavan, When optimism hurts: inflated predictions in psychiatric neuroimaging. Biol. Psychiatry, 2014. 75(9): p. 746748.

34. Wager, T.D., et al., An fMRI-based neurologic signature of physical pain. N Engl J Med, 2013. 368(15): p. $1388-97$.

35. Lindquist, M.A., et al., Group-regularized individual prediction: theory and application to pain. Neuroimage, 2015.

36. Krishnan, A., et al., Somatic and vicarious pain are represented by dissociable multivariate brain patterns. eLife, 2016.

37. Fu, C.H., et al., Pattern classification of sad facial processing: toward the development of neurobiological markers in depression. Biol Psychiatry, 2008. 63(7): p. 656-62.

38. Kassam, K.S., et al., Identifying Emotions on the Basis of Neural Activation. Plos One, 2013. 8(6).

39. Wager, T.D., et al., An fMRI-based neurologic signature of physical pain. The New England Journal of Medicine, 2013. 368(15): p. 13881397. 
40. Woo, C.W., et al., Separate neural representations for physical pain and social rejection. Nature communications, 2014 . 5: p. 5380.

41. Wager, T.D. and C.W. Woo, fMRI in analgesic drug discovery. Sci Transl Med, 2015. 7(274): p. $274 \mathrm{fs} 6$.

42. Woo, C.W., et al., Quantifying cerebral contributions to pain beyond nociception. Nature Communications, 2016.

43. Brodersen, K.H., et al., Decoding the perception of pain from fMRI using multivariate pattern analysis. Neuroimage, 2012. 63(3): p. 116270.

44. Horikawa, T., et al., Neural decoding of visual imagery during sleep. Science, 2013. 340(6132): p. 639-42.

45. Kamitani, Y. and F. Tong, Decoding the visual and subjective contents of the human brain. Nat Neurosci, 2005. 8(5): p. $679-85$.

46. Kay, K.N., et al., Identifying natural images from human brain activity. Nature, 2008. 452(7185): p. $352-355$.

47. Huth, A.G., et al., A continuous semantic space describes the representation of thousands of object and action categories across the human brain. Neuron, 2012. 76(6): p. 1210-24.

48. Haxby, J.V., A.C. Connolly, and J.S. Guntupalli, Decoding Neural Representational Spaces Using Multivariate Pattern Analysis. Annual Review of Neuroscience, 2014. 37(1).

49. Haxby, J.V., et al., Distributed and overlapping representations of faces and objects in ventral temporal cortex. Science, 2001. 293(5539): p. 2425-30.

50. Haxby, J.V., et al., A common, high-dimensional model of the representational space in human ventral temporal cortex. Neuron, 2011. 72(2): p. 404-16.

51. Rissman, J., H.T. Greely, and A.D. Wagner, Detecting individual memories through the neural decoding of memory states and past experience. Proceedings of the National Academy of Sciences of the United States of America, 2010. 107(21): p. 9849-9854.

52. Uncapher, M.R., et al., Goal-Directed Modulation of Neural Memory Patterns: Implications for fMRI-Based Memory Detection. J Neurosci, 2015. 35(22): p. 8531-45.

53. Cukur, T., et al., Attention during natural vision warps semantic representation across the human brain. Nat Neurosci, 2013. 16(6): p. 763-70.

54. Rosenberg, M.D., et al., A neuromarker of sustained attention from whole-brain functional connectivity. Nat Neurosci, 2016. 19(1): p. 165-71.

55. Shinkareva, S.V., et al., Using fMRI Brain Activation to Identify Cognitive States Associated with Perception of Tools and Dwellings. Plos One, 2008. 3(1).

56. Huth, A.G., et al., Decoding the Semantic Content of Natural Movies from Human Brain Activity. Front Syst Neurosci, 2016. $10:$ p. 81.

57. Eisenbarth, H., L.J. Chang, and T.D. Wager, Multivariate Brain Prediction of Heart Rate and Skin Conductance Responses to Social Threat. J Neurosci, 2016. 36(47): p. 11987-11998.

58. Kragel, P.A., et al., Representation, Pattern Information, and Brain Signatures: From Neurons to Neuroimaging. Neuron, 2018. 99(2): p. 257-273.

59. Reddan, M.C., M.A. Lindquist, and T.D. Wager, Effect Size Estimation in Neuroimaging. JAMA Psychiatry, 2016.

60. Gabrieli, J.D.E., S.S. Ghosh, and S. Whitfield-Gabrieli, Prediction as a humanitarian and pragmatic contribution from human cognitive neuroscience. Neuron, 2015. 85(1): p. 11-26.

61. Davis, K.D., et al., Discovery and validation of biomarkers to aid the development of safe and effective pain therapeutics: challenges and opportunities. Nat. Rev. Neurol., 2020. 16(7): p. 381-400.

62. Group, F.-N.B.W., BEST (Biomarkers, EndpointS, and other Tools) Resource. 2016, Silver Spring (MD): Food and Drug Administration (US).

63. Knutson, B. and A. Genevsky, Neuroforecasting Aggregate Choice. Curr. Dir. Psychol. Sci., 2018. 27(2): p. 110-115.

64. Volkow, N.D., G. Koob, and R. Baler, Biomarkers in substance use disorders. ACS chemical neuroscience, 2015. 6(4): p. $522-525$.

65. Volkow, N.D. and R.D. Baler, Brain imaging biomarkers to predict relapse in alcohol addiction. JAMA psychiatry, 2013. 70(7): p. 661-663.

66. Steele, V.R., et al., Machine Learning of Functional Magnetic Resonance Imaging Network Connectivity Predicts Substance Abuse Treatment Completion. Biol Psychiatry Cogn Neurosci Neuroimaging, 2018. 3(2): p. 141-149.

67. Noori, H.R., A.C. Linan, and R. Spanagel, Largely overlapping neuronal substrates of reactivity to drug, gambling, food and sexual cues: $A$ comprehensive meta-analysis. European Neuropsychopharmacology, 2016. 26(9): p. 1419-1430.

68. Volkow, N.D., R.A. Wise, and R. Baler, The dopamine motive system: Implications for drug and food addiction. Nature Reviews Neuroscience, 2017. 18(12): p. 741-752.

69. Volkow, N.D., et al., The addictive dimensionality of obesity. Biol Psychiatry, 2013. 73(9): p. 811-8.

70. Volkow, N.D., et al., Obesity and addiction: neurobiological overlaps. Obesity Reviews, 2013. 14(1): p. 2-18. 
71. Tang, D.W., et al., Food and drug cues activate similar brain regions: A meta-analysis of functional MRI studies. Physiol Behav, 2012. 106: p. 317-324.

72. Ziauddeen, H. and P.C. Fletcher, Is food addiction a valid and useful concept? Obesity Reviews, 2013. 14(1): p. 19-28.

73. Ziauddeen, H., I.S. Farooqi, and P.C. Fletcher, Obesity and the brain: how convincing is the addiction model? Nat Rev Neurosci, 2012. 13(4): p. 279-86.

74. Suzuki, S., et al., Regulation of Craving and Negative Emotion in Alcohol Use Disorder. Biological Psychiatry: Cognitive Neuroscience and Neuroimaging, 2020. 5(2): p. 239-250.

75. Schafer, M., M.N. Potenza, and H. Kober, Neural Mechanisms underlying regulation of craving in Cocaine Dependence. in prep.

76. Kober, H., et al., Prefrontal-striatal pathway underlies cognitive regulation of craving. Proceedings of the National Academy of Sciences, 2010. 107(33): p. 14811-14816

77. Wager, T.D., et al., Predicting individual differences in placebo analgesia: contributions of brain activity during anticipation and pain experience. J. Neurosci., 2011. 31(2): p. 439-452.

78. Chang, L.J., et al., A Sensitive and Specific Neural Signature for Picture-Induced Negative Affect. PLoS Biol, 2015. 13(6): p. e1002180.

79. King, K.M., et al., Marker or mediator? The effects of adolescent substance use on young adult educational attainment. Addiction, 2006. 101(12): p. 1730-1740.

80. Cosme, D., et al., Multivariate neural signatures for health neuroscience: Assessing spontaneous regulation during food choice. Soc. Cogn. Affect. Neurosci., 2020.

81. Wilcox, C.E., M.E. Brett, and V.D. Calhoun, Objective markers for psychiatric decision-making: How to move imaging into clinical practice. Neuroimage Clin, 2020. 26: p. 102084.

82. Potenza, M.N., et al., Neuroscience of Behavioral and Pharmacological Treatments for Addictions. Neuron, 2011. 69(4): p. 695-712.

83. Bartra, O., J.T. McGuire, and J.W. Kable, The valuation system: a coordinate-based meta-analysis of BOLD fMRI experiments examining neural correlates of subjective value. Neuroimage, 2013. 76: p. 412-427.

84. Kable, J.W. and P.W. Glimcher, The neural correlates of subjective value during intertemporal choice. Nat. Neurosci., 2007. 10(12): p. 1625-1633.

85. Kühn, S. and J. Gallinat, Common biology of craving across legal and illegal drugs-a quantitative meta-analysis of cue-reactivity brain response. Eur. J. Neurosci., 2011.

86. Chase, H.W., et al., The neural basis of drug stimulus processing and craving: an activation likelihood estimation meta-analysis. Biol. Psychiatry, 2011. 70(8): p. 785-793.

87. Engelmann, J.M., et al., Neural substrates of smoking cue reactivity: A meta-analysis of fMRI studies. Neuroimage, 2012. 60(1): p. 252262.

88. Schacht, J.P., R.F. Anton, and H. Myrick, Functional neuroimaging studies of alcohol cue reactivity: a quantitative meta-analysis and systematic review. Addict. Biol., 2013. 18(1): p. 121-133.

89. Noori, H.R., A. Cosa Linan, and R. Spanagel, Largely overlapping neuronal substrates of reactivity to drug, gambling, food and sexual cues: A comprehensive meta-analysis. Eur. Neuropsychopharmacol., 2016. 26(9): p. 1419-1430.

90. Lin, X., et al., Neural substrates of smoking and reward cue reactivity in smokers: a meta-analysis of fMRI studies. Transl. Psychiatry, 2020. 10(1): p. 97.

91. Naqvi, N.H., et al., The insula: a critical neural substrate for craving and drug seeking under conflict and risk. Annals of the New York Academy of Sciences, 2014. 1316(1): p. 53-70.

92. Naqvi, N.H., et al., Damage to the insula disrupts addiction to cigarette smoking. Science, 2007. 315(5811): p. 531-534.

93. Garavan, H., Insula and drug cravings. Brain Structure and Function, 2010. 214(5-6): p. 593-601.

94. Craig, A.D., How do you feel-now? The anterior insula and human awareness. Nature reviews. Neuroscience, $2009.10(1)$ : p. 59.

95. Naqvi, N.H. and A. Bechara, The hidden island of addiction: the insula. Trends in Neurosciences, 2009. 32(1): p. 56-67.

96. de Araujo, I.E., M. Schatzker, and D.M. Small, Rethinking Food Reward. Annu. Rev. Psychol., 2020. 71: p. $139-164$.

97. Woo, C.-W., et al., Building better biomarkers: brain models in translational neuroimaging Nature Neuroscience. Nat. Neurosci., 2017. 20(3): p. 365-377.

98. Lopez, R.B., et al., Boundary conditions of methamphetamine craving. Experimental and Clinical Psychopharmacology, 2015. 23(6): p. 436-444.

99. Naqvi, N.H., et al., Cognitive regulation of craving in alcohol-dependent and social drinkers. Alcoholism: Clinical and Experimental Research, 2015. 39(2): p. 343-349. 
100. Boswell, R.G., et al., Training in Cognitive Strategies Reduces Eating and Improves Food Choice. Proceedings of the National Academy of Sciences (PNAS), 2018. 115(48): p. E11238-E11247.

101. Tibshirani, R., Regression shrinkage and selection via the lasso. Journal of the Royal Statistical Society. Series B (Methodological), 1996 : p. 267-288.

102. Glasser, M.F., et al., A multi-modal parcellation of human cerebral cortex. Nature, 2016. 536(7615): p. 171-178.

103. Pauli, W.M., et al., Regional specialization within the human striatum for diverse psychological functions. Proc. Natl. Acad. Sci. U. S. A., 2016. 113(7): p. 1907-1912.

104. Diedrichsen, J., et al., A probabilistic MR atlas of the human cerebellum. Neuroimage, 2009. 46(1): p. 39-46.

105. Schaefer, A., et al., Local-Global Parcellation of the Human Cerebral Cortex from Intrinsic Functional Connectivity MRI. Cereb. Cortex, 2018. 28(9): p. 3095-3114.

\section{Tables}

1. Clusters of FDR-corrected bootstrapped weights of the NCS.

\begin{tabular}{|c|c|c|c|c|c|c|c|}
\hline Region name & Vol $\left(\mathrm{mm}^{3}\right)$ & $\mathbf{x}$ & $\mathbf{Y}$ & $\mathbf{z}$ & $\max (\mathrm{Z})$ & Atlas region (see note) & Large-scale network / structure (see note) \\
\hline \multicolumn{8}{|l|}{ Positive weights } \\
\hline Postcentral gyrus / somatosensory cortex & 513 & -42 & -27 & 60 & 4.74 & $3 b_{-} L \mathrm{~L}$ & SomatomotorA \\
\hline Inferior temporal gyrus & 162 & 57 & -51 & -15 & 4.62 & TE1p_R & Fronto_ParietalB \\
\hline Subcallosal gyrus / ventral striatum & 81 & 12 & 9 & -21 & 4.47 & $\overline{\text { pOFC_R }}$ & Limbic \\
\hline Cerebellum & 162 & -48 & -66 & -42 & 4.42 & CrusI_L & Cerebellum \\
\hline Superior frontal gyrus / dlPFC & 216 & -24 & 33 & 57 & 4.36 & s6_8_L & Default_ModeA \\
\hline Inferior parietal lobule & 108 & 30 & -63 & 45 & 4.30 & IP1_R & Dorsal_AttentionA \\
\hline Rostral gyrus / vmPFC & 81 & -6 & 51 & 3 & 4.23 & $9 \mathrm{~m}_{-} \mathrm{L}$ & Default_ModeB \\
\hline Supraparietal lobule & 54 & -12 & -60 & 60 & 4.17 & 7Am_L & Dorsal_AttentionB \\
\hline Retrosplenial cortex & 27 & -3 & -54 & 15 & 4.07 & v23ab_L & Default_ModeA \\
\hline Thalamus & 108 & 0 & -9 & 6 & 4.07 & Thal_MD & Diencephalon \\
\hline Angular gyrus & 27 & 57 & -36 & 21 & 4.00 & PSL_R & Temporal_Parietal \\
\hline Paracentral lobule / SMA & 54 & -3 & -6 & 51 & 3.99 & $24 d d \_L$ & SomatomotorA \\
\hline Supramarginal gyrus & 27 & 57 & -33 & 45 & 3.96 & PF_R & Ventral_AttentionA \\
\hline Lateral occipital & 27 & 51 & -72 & -18 & 3.94 & PH_R & Dorsal_AttentionA \\
\hline Anterior midcingulate gyrus (aMCC) & 27 & -6 & 36 & 18 & 3.90 & p24_L & Default_ModeA \\
\hline \multicolumn{8}{|l|}{ Negative weights } \\
\hline Postcentral gyrus / somatosensory cortex & 1917 & -51 & -18 & 51 & -7.37 & 3b_L & SomatomotorA \\
\hline Middle temporal gyrus & 243 & 54 & -63 & 6 & -4.77 & TPOJ2_R & Dorsal_AttentionA \\
\hline Angular gyrus & 162 & 42 & -60 & 33 & -4.43 & PGi_R & Default_ModeC \\
\hline Visual cortex & 81 & 39 & -72 & -18 & -4.26 & PIT_R & Visual_Central \\
\hline Superior temporal gyrus & 27 & 54 & -3 & 0 & -4.20 & PBelt_R & SomatomotorB \\
\hline Visual cortex & 27 & 0 & -84 & 6 & -4.12 & V1_R & Visual_Peripheral \\
\hline Superior occipital gyrus & 54 & 18 & -84 & 45 & -4.03 & V6A_R & Visual_Peripheral \\
\hline Precuneus & 27 & 9 & -63 & 33 & -3.99 & POS2_R & Fronto_ParietalC \\
\hline Superior parietal lobule & 54 & -12 & -57 & 75 & -3.96 & 7AL_L & Dorsal_AttentionB \\
\hline Angular gyrus & 27 & 57 & -63 & 33 & -3.90 & No label & No description \\
\hline
\end{tabular}

Note. Significant positive and negative weights contributing to the NCS (FDR corrected $q<0.05$ across the whole brain graymatter mask). Cortical atlas regions (Ctx) are labeled based on the multimodal cortical parcellation by Glasser et al., [102], basal ganglia regions are based on Pauli et al. [103], cerebellar regions on Diedrichsen, et al. [104], and brainstem regions based on a combination of studies [103]. Large-scale network names are based on the resting-state parcellation by Schaefer et al. [105]. The entire combined anatomical atlas is available on Github: https://github.com/canlab/Neuroimaging_Pattern_Masks/tree/master/Atlases_and_parcellations/2018_Wager_combined_atlas. This repository includes multiple atlases and other meta-analytic and multivariate maps. Tools for manipulating and analyzing this and other atlases are in the CANlab Core Tools repository: https://github.com/canlab/CanlabCore.

Table 2. Predictive performance of the NCS and effect sizes for each study sample. 


\begin{tabular}{|c|c|c|c|c|c|}
\hline Dataset & Cues & Sample & $\mathbf{N}$ & Prediction-outcome (glmfit_multilevel) & Effect size Cohen's $d$ \\
\hline Study $1 \mathrm{a}$ & Cigarette and food cues & Cigarette smokers & 21 & $\begin{array}{l}\text { beta }=0.22, \mathrm{STE}=0.09 \\
t(20)=2.53, p=0.020\end{array}$ & $d=0.56$ \\
\hline Study $1 \mathrm{~b}$ & Cigarette and food cues & Non-smokers & 24 & $\begin{array}{l}\text { beta }=0.37, \mathrm{STE}=0.07 \\
t(23)=5.12, p<0.001\end{array}$ & $d=1.07$ \\
\hline Study 2 & Alcoholic drinks and food cues & Alcohol users & 17 & $\begin{array}{l}\text { beta }=0.31, \mathrm{STE}=0.12 \\
t(16)=2.63, p=0.018\end{array}$ & $d=0.66$ \\
\hline Study 3a & Cocaine and food cues & Cocaine users & 21 & $\begin{array}{l}\text { beta }=0.36, \text { STE }=0.10 \\
t(20)=3.72, p=0.001\end{array}$ & $d=0.83$ \\
\hline Study 3b & Cocaine and food cues & Non-users & 18 & $\begin{array}{r}\text { beta }=0.56, \mathrm{STE}=0.10 \\
t(17)=5.80, p<0.001\end{array}$ & $d=1.41$ \\
\hline All Studie & & & 101 & $\begin{array}{r}\text { beta }=0.36, \text { STE }=0.04 \\
t(100)=8.59, p<0.001\end{array}$ & $d=0.86$ \\
\hline
\end{tabular}

3. Participant demographics by group and study cohort.

\begin{tabular}{|c|c|c|c|c|}
\hline Study & & Substance Users & Non-Users & Statistics ( $p$-value) \\
\hline 1 & $\begin{array}{l}\mathbf{N} \\
\text { Mean age } \\
\text { Male } \\
\text { Female } \\
\text { White } \\
\text { Black } \\
\text { Asian } \\
\text { Education (years) }\end{array}$ & \begin{tabular}{|c|}
17 \\
33.4 \\
10 \\
7 \\
8 \\
8 \\
1 \\
14.3 \\
\end{tabular} & - & \\
\hline 2 & $\begin{array}{l}\mathbf{N} \\
\text { Mean age } \\
\text { Male } \\
\text { Female } \\
\text { White } \\
\text { Black } \\
\text { Education (years) } \\
\end{array}$ & $\begin{array}{c}21 \\
43.5 \\
18 \\
3 \\
6 \\
14 \\
12.6 \\
\end{array}$ & $\begin{array}{c}\mathbf{1 8} \\
\mathbf{4 2 . 1} \\
12 \\
6 \\
7 \\
11 \\
15.3 \\
\end{array}$ & $\begin{array}{c}0.441 \\
0.251 \\
0.551 \\
2.4 \mathrm{E}-05^{*}\end{array}$ \\
\hline 3 & $\begin{array}{l}\mathbf{N} \\
\text { Mean age } \\
\text { Male } \\
\text { Female } \\
\text { White } \\
\text { Black } \\
\text { Asian } \\
\text { Native American } \\
\text { Other } \\
\text { Education (years) }\end{array}$ & $\begin{array}{c}21 \\
26.8 \\
13 \\
8 \\
11 \\
1 \\
5 \\
1 \\
3 \\
15.2 \\
\end{array}$ & $\begin{array}{c}24 \\
26.9 \\
14 \\
10 \\
13 \\
2 \\
5 \\
0 \\
4 \\
15.7 \\
\end{array}$ & $\begin{array}{l}0.980 \\
0.807\end{array}$ \\
\hline Summary & $\begin{array}{l}\mathbf{N} \\
\text { Mean age } \\
\text { Male } \\
\text { Female } \\
\text { White } \\
\text { Black } \\
\text { Asian } \\
\text { Native American } \\
\text { Other } \\
\text { Education (years) }\end{array}$ & $\begin{array}{c}59 \\
34.6 \\
41 \\
18 \\
25 \\
23 \\
6 \\
1 \\
3 \\
14.0 \\
\end{array}$ & $\begin{array}{c}42 \\
33.4 \\
26 \\
16 \\
20 \\
13 \\
5 \\
0 \\
4 \\
15.5 \\
\end{array}$ & $\begin{array}{l}0.566 \\
0.519\end{array}$ \\
\hline
\end{tabular}

Note. Participants were matched for age, sex, and race. Cocaine users had significantly lower educational attainment.

\section{Figures}


a Regulation of Craving Task design

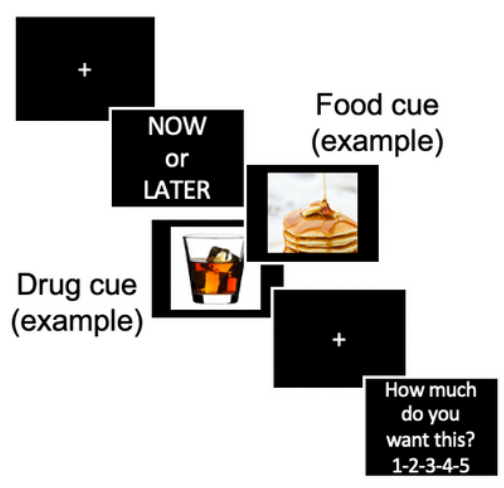

\section{b Study samples}

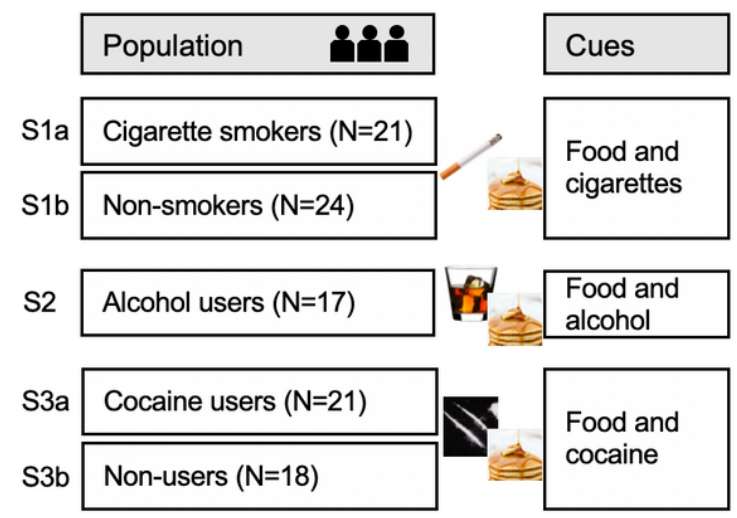

\section{d fMRI-based prediction of craving}

\section{fMRI data by rating level across cue type and regulation condition}

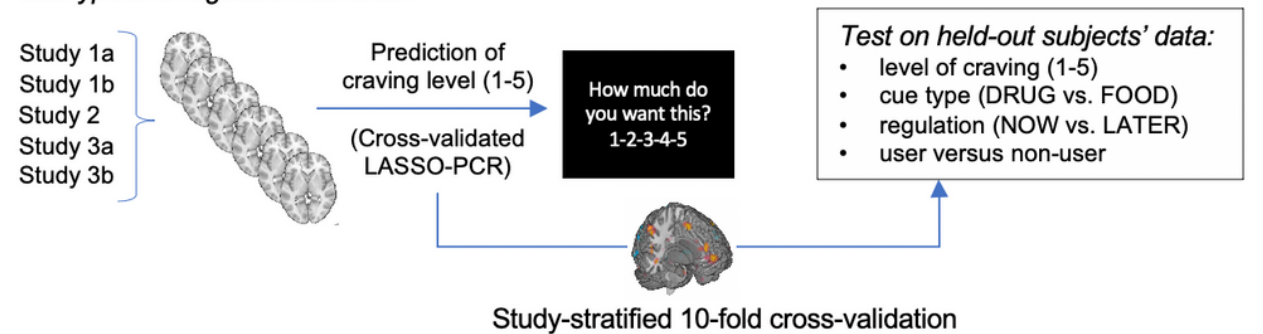

\section{d Permutation test results}
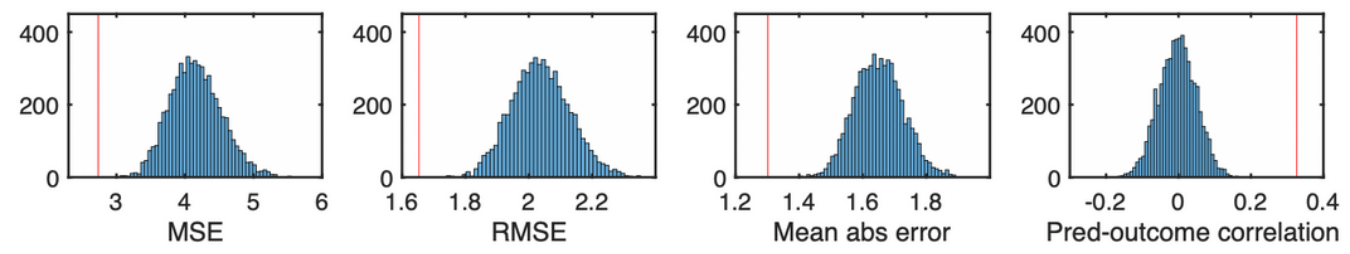

Figure 1

Study design and analytic approach. (a) In the Regulation of Craving (ROC) task, participants were presented with a series of photographs depicting either drugs (alcoholic drinks, cocaine, or cigarettes,) or highly palatable food items. Before presentation of the cues, participants were instructed ( $2 \mathrm{~s}$ written cue) to either consider the immediate consequences of consumption of the items ('NOW' condition), or their negative (typically long-term) consequences ('LATER' condition). At the end of each trial, participants rated their craving ('How much do you want this?'), using a 1-5 Likert scale. (b) The present study employed the pooled data from 3 previous studies (5 groups of participants). Study 1 tested the ROC task (displaying cigarette and food cues) in 21 heavy smokers (Study 1a) and 24 non-smokers (Study 1b). Study 2 tested the ROC task (displaying alcohol and food cues) in participants fulfilling diagnostic criteria of alcohol use disorder ( $N=17$, see details in methods and [74]). Study 3 tested the ROC task (displaying cocaine and food cues) in 21 individuals with cocaine use disorder (Study 3a) and 18 matched non-users (Study 3b). (c) For each participant from all five studies, we computed brain activation images (beta-estimates) for each level of craving (1-5). These images were then used in a LASSO-PCR (least absolute shrinkage and selection operator - principal component regression) machine-learning algorithm to predict level of craving (1-5) based on brain activity. Cross-validation (ten folds stratified for studies and participants population) allowed assessment of (a) predictive accuracy of the pattern for craving, (b) whether it was differentially activated for drug versus food cues, and (c) whether it was differentially activated for the two regulation conditions (NOW vs. LATER), and (d) whether the pattern can differentiate drug users from non-users. (d) Permutation test results. Null distributions are plotted in blue bars, observed accuracy measures as red lines. For all measures, none of the permutation samples performed as well as the observed results (all p's $<0.002$ ). 
a
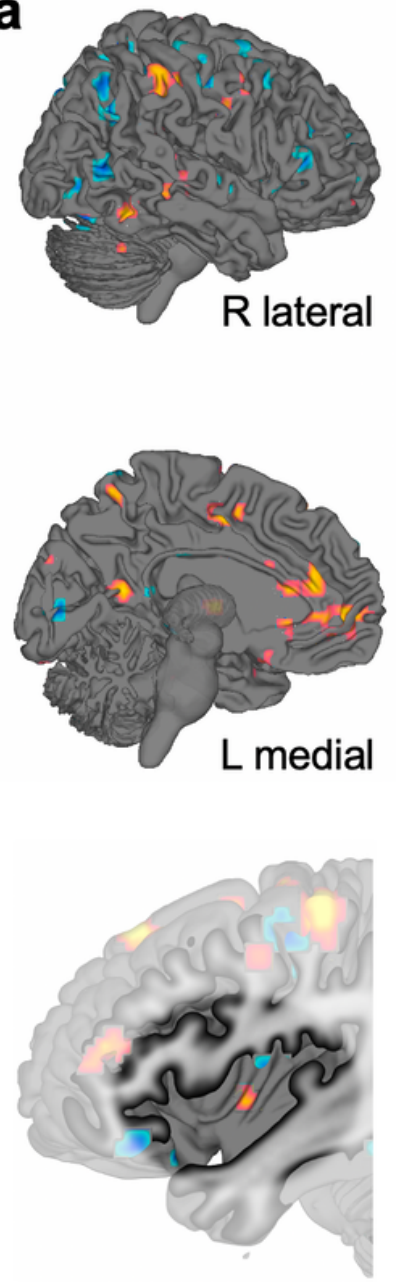

L insula
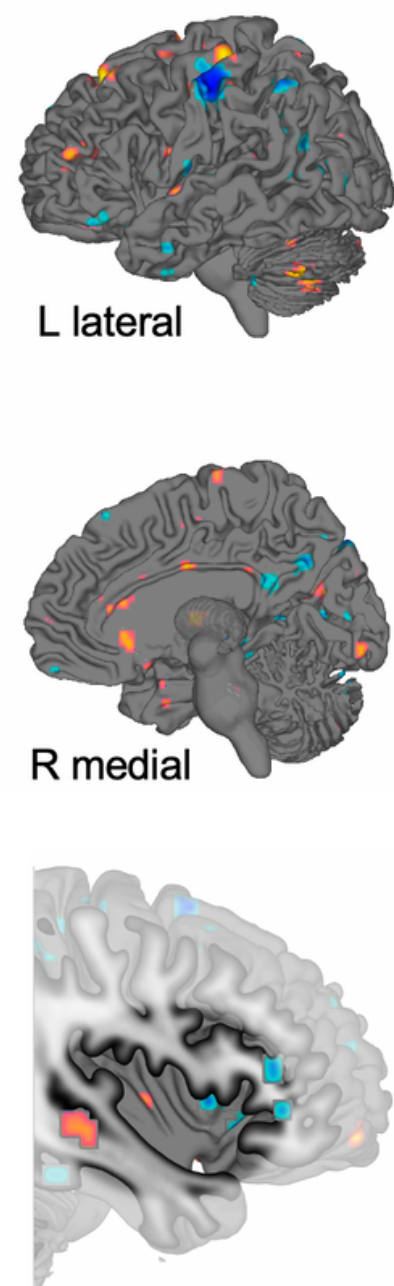

$\mathrm{R}$ insula b

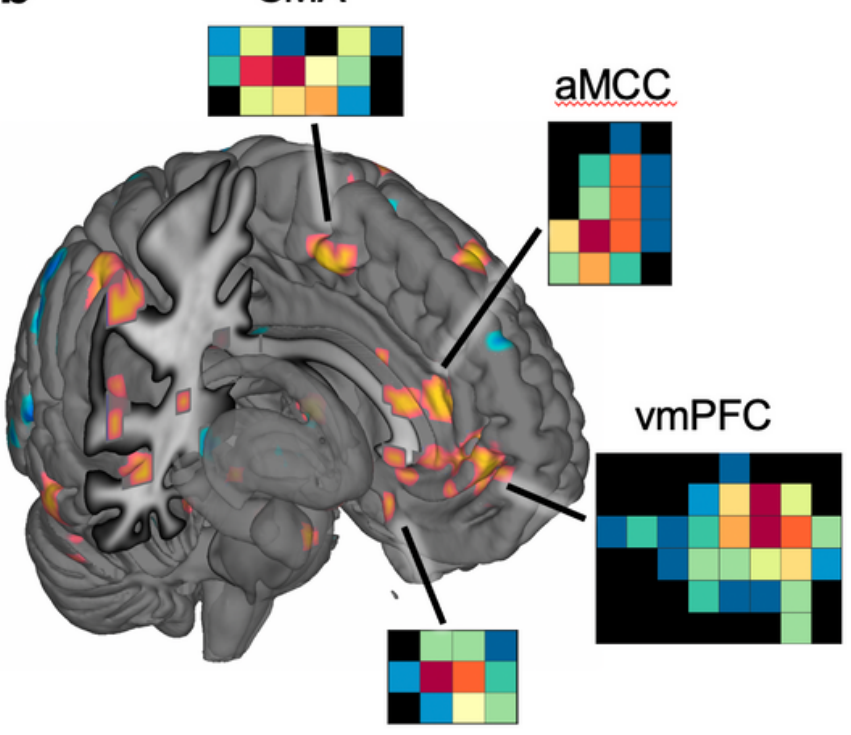

sgACC/vS

positive weights

$p<0.000002$

$p<0.005$

$p<0.005$

$p=1.7 \mathrm{e}-13$

negative weights

Figure 2

Thresholded display of the NCS. Note that unthresholded patterns are used for prediction; this thresholded pattern is for illustration purposes at $p<0.005$ uncorrected. (a) Medial, lateral, and insula displays of the most consistent pattern weights. (b) Pop-out rectangles show the multivariate pattern for selected clusters of interest. Warm (yellow-red) color indicates positive weights, cold (cyan-purple) color indicates negative weights in predicting drug and food craving. P-values are based on bootstrapping and indicate the areas that contribute most consistently with positive or negative weights. See Table 1 for a list of FDR corrected weights. 
a

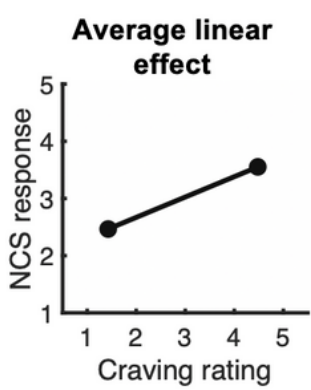

Individual slopes

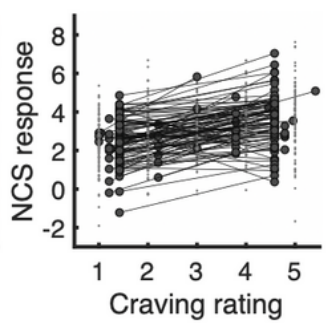

b

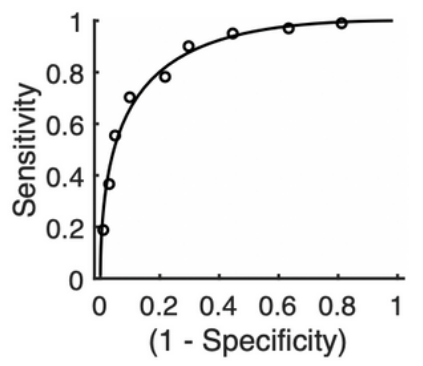

C
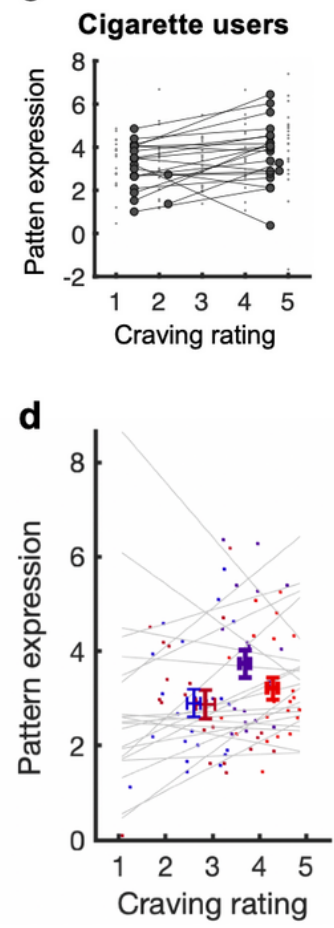
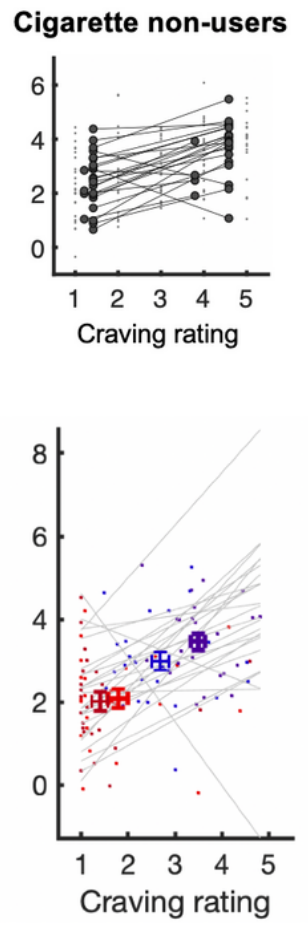
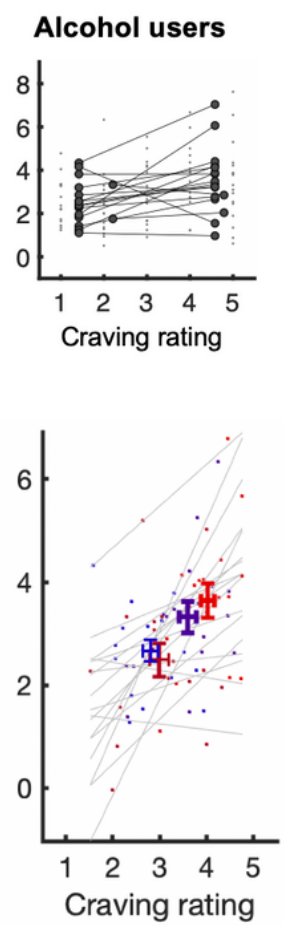
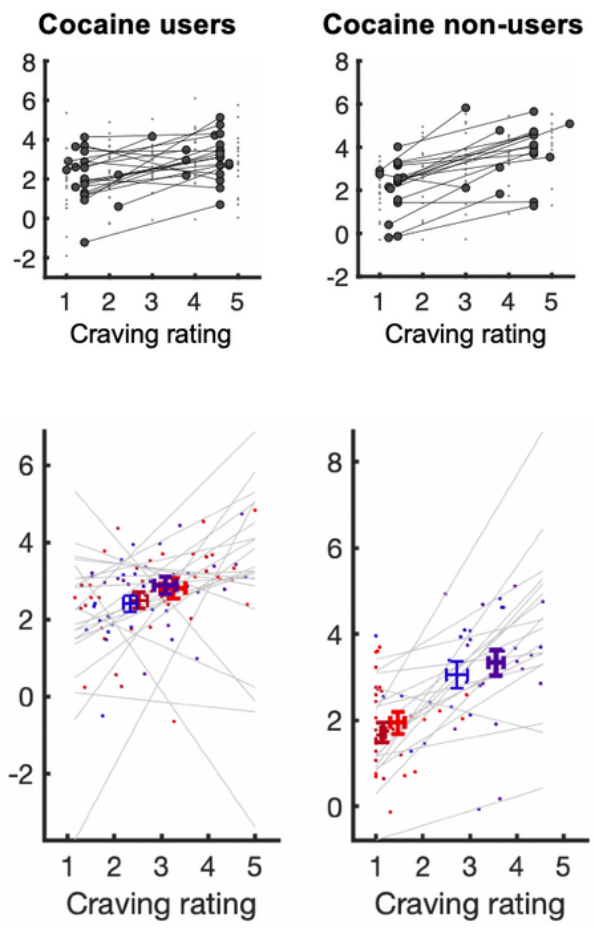

$$
\text { + Drug NOW + Drug LATER + Food NOW + Food LATER }
$$

\section{Figure 3}

Predictive performance of the NCS. (a) Relationship between behavioral craving levels (binned into 5 levels) and NCS-predicted craving levels across all participants. Individual data points and slopes are shown on the right-hand panel. (b) Receiver-operating characteristic (ROC) plot for the prediction of highest versus lowest levels of craving (forced-choice discrimination). (c) Individual datapoints and slopes for the relationship between craving levels and NCS for all five datasets (significant positive association in each study). (d) Average levels of craving ratings (on the $x$-axis) and NCS responses (on the $y$-axis) for each of the four experimental conditions (drug versus food cues, NOW versus LATER instruction) and within each dataset. Gray lines show individual slopes across the four conditions. Dots indicate individual data points for each condition and participant. Error bars indicate standard errors of the mean (SE). 
a

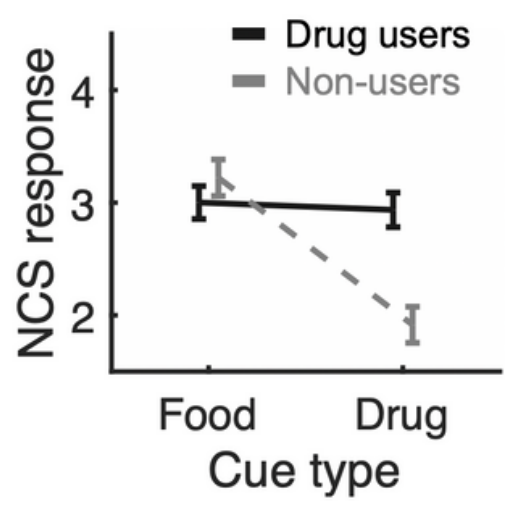

b

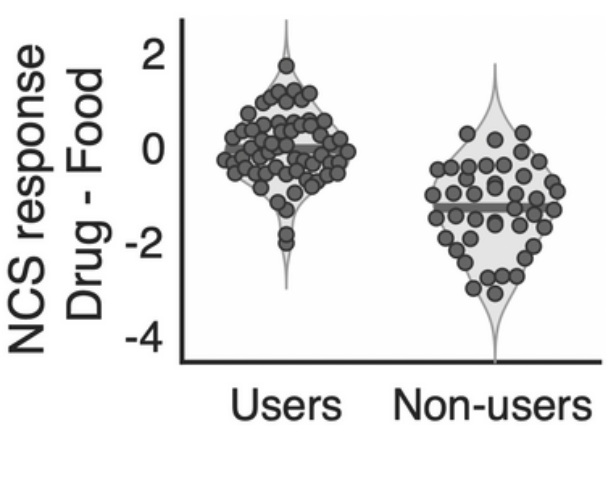

C

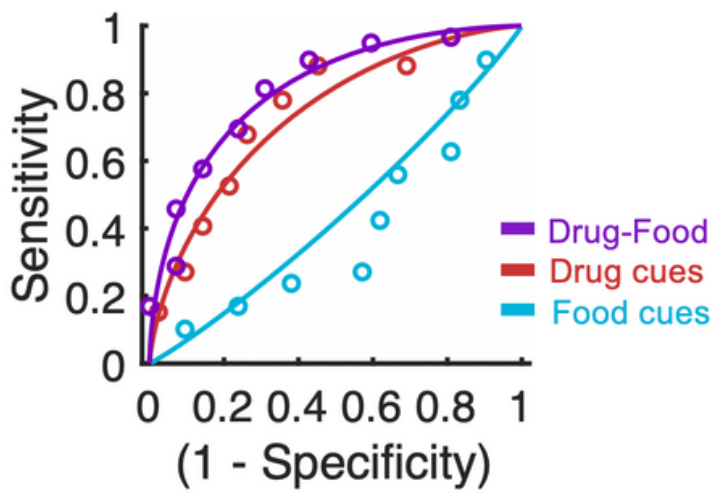

\section{Figure 4}

Classification of drug users versus non-users based on NCS responses to drugs and food. (a) Out-of-sample responses of the NCS to drug and food cues, in drug users and non-users. Error bars indicate SE. (b) Differences in NCS responses to drug minus food cues, in drug users versus non-users. (c) Response-operating characteristic plots for NCS-based prediction of drug use. Whereas NCS responses to food cues did not significantly separate users from non-users (in blue), NCS responses to drug cues were able to significantly separate users from nonusers (in red). Differential NCS responses to drug-food cues (in purple) had the highest classification accuracy ( $77 \%$, see text for details).

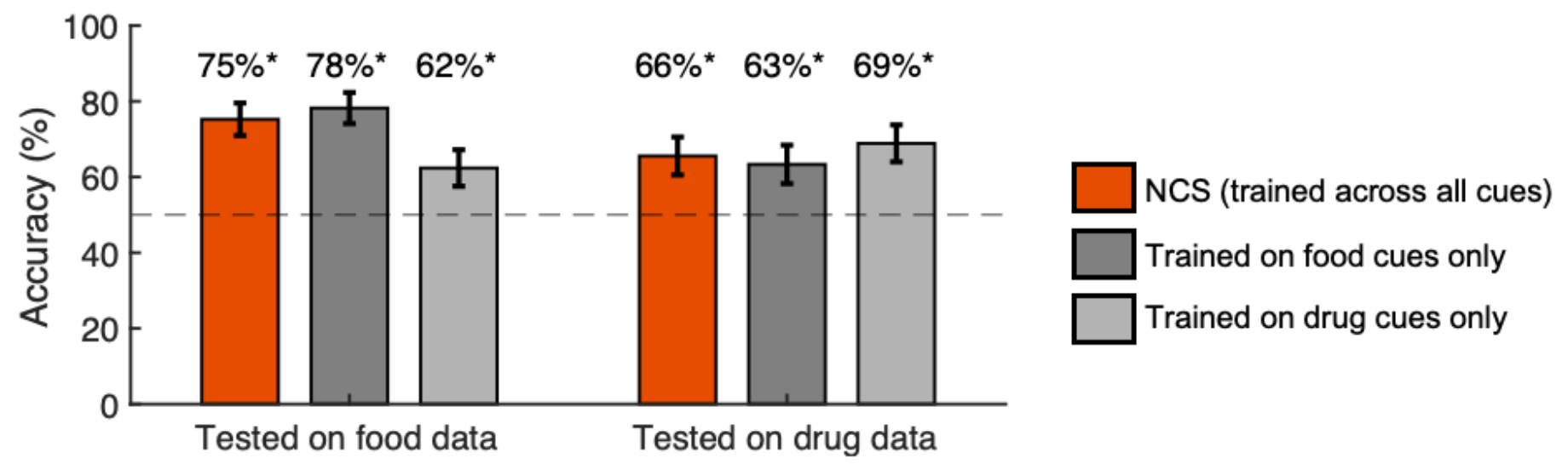

Figure 5

Cross-prediction of drug and food craving based on drug- and food-based brain patterns. Compared to the NCS (trained across all conditions, red bars), training on drug or food cues separately (in gray) does not improve accuracy, suggesting shared predictive patterns for craving of drug and food cues. Error bars indicate SE. 